Faculty of Economics and Social Sciences

Department of Economics

\title{
Maximal Domains for Strategy-proof or Maskin monotonic Choice Rules
}

Olivier Bochet

Ton Storcken

09-01

August 2008

\section{DISCUSSION PAPERS}




\title{
Maximal Domains for Strategy-proof or Maskin monotonic Choice Rules* Olivier Bochet ${ }^{\dagger}$ and Ton Storcken ${ }^{\ddagger}$
}

\author{
August 2008
}

\begin{abstract}
Domains of individual preferences for which the well-known impossibility Theorems of Gibbard-Satterthwaite and Muller-Satterthwaite do not hold are studied. First, we introduce necessary and sufficient conditions for a domain to admit non-dictatorial, Pareto efficient and either strategy-proof or Maskin monotonic social choice rules. Next, to comprehend the limitations the two Theorems imply for social choice rules, we search for the largest domains that are possible. Put differently, we look for the minimal restrictions that have to be imposed on the unrestricted domain to recover possibility results. It turns out that, for such domains, the conditions of inseparable pair and of inseparable set yield the only maximal domains on which there exist non-dictatorial, Pareto efficient and strategy-proof social choice rules. Next, we characterize the maximal domains which allow for Maskin monotonic, non-dictatorial and Pareto-optimal social choice rules.
\end{abstract}

\section{Introduction}

The two most negative results on the decentralization of social choice rules (henceforth, rules) are, respectively, the Gibbard-Satterthwaite Theorem (Gib-

${ }^{*}$ Corresponding author: olivier.bochet@vwi.unibe.ch.

${ }^{\dagger}$ University of Bern and Maastricht University.

${ }_{\ddagger}^{\ddagger}$ Maastricht University. 
bard (1973) and Satterthwaite (1975)) and the Muller-Satterthwaite Theorem (Muller and Satterthwaite, 1977) theorems. ${ }^{1}$ The GS Theorem states that over an unrestricted domain of linear orderings-and with at least three alternatives- any surjective and strategy-proof rule is dictatorial. On the other hand, the MS Theorem state that any unanimous and Maskin monotonic is dictatorial. ${ }^{2}$

It is by now well-understood that the two aforementioned Theorems strongly rely on the assumption of an unrestricted preference domain. Restricted domains have delivered possibility results on strategy-proof and Maskin monotonic rules. A striking example is the single-peaked preference domain (see e.g. Moulin, 1980) for which there exists a huge class of strategy-proof rules called generalized median voting rules. ${ }^{3,4}$ Therefore, on the one hand (i) there is a misperception concerning the robustness of the GS and MS Theorems, and on the other hand (ii) often the gap between possibility and impossibility results is not fully understood. This paper is an attempt to provide some answers - at least partially- to (i) and (ii) above. In order to do so, we address two fundamental questions. The first one is to know when a preference domain escapes the negative conclusions of the aforementioned theorems with non-trivial rules -i.e. rules that are non-dictatorial, Pareto efficient and strategy-proof/Maskin monotonic. Stated differently, we want to know when a preference domain is a strategy-proof/Maskin monotonicity possibility domain. The two necessary and sufficient conditions we uncover are respectively strategy-proof admissibility and Maskin admissibility. They can be readily checked for any preference domain at hand. Next, the second question is to check how much restrictions are needed from the unrestricted domain to recover a possibility domain. Another way to tackle this question is the following: how many preference profiles should be removed from the unrestricted domain in order to get a possibility domain? Therefore, we look for such domains which are maximal -i.e. there are no supersets of this do-

\footnotetext{
${ }^{1}$ Henceforth, we refer to the Gibbard-Satterthwaite Theorem and the MullerSatterthwaite Theorem as GS and MS Theorems respectively.

${ }^{2}$ Strategy-proofness and Maskin monotonicity are, respectively, necessary conditions for dominant strategy implementation and Nash implementation. Thus restriction to normal game forms and uniqueness of dominant strategy/Nash equilibrium outcomes induce that only trivial rules can be decentralized.

${ }^{3}$ The class of Maskin monotonic rules on such a domain is somewhat smaller than the class of strategy-proof rules. See for instance Bochet and Klaus (2008)

${ }^{4}$ There is a vast literature providing possibility results in various models. We voluntarily abstract from an extensive overview.
} 
main on which such rules exist. Such maximal domains indicate a minimal necessary restriction of the set of profiles and herewith the impact of the socalled impossibility theorems mentioned above: the smaller these maximal domains are the more restrictive the properties of the social choice rules of these impossibility theorems are. The necessary and sufficient conditions we uncover are -at least for the strategy-proof case- easy to interpret and check.

We should point out that we are indeed not the first one to address the question of the maximality of domains -at least for the strategy-proof casebut we depart from the "standard" approach. In the literature, the approach that is usually followed is to take a "possibility" domain and to find the maximal enlargement of this domain so that the possibility result still hold. For instance, this is the case of Barberà, Sonnenschein and Zhou (1994); Barberà, Gul and Stacchetti (1994); Serizawa (1995); Serizawa and Ching (1998); Berga and Serizawa (2000), or Masso and Neme (2001). Each paper deals with entire restricted domain of preferences. Recently, Puppe and Tasńdi (2006) study maximal domains over which the Borda count satisfies Maskin monotonicity. These domains are called cyclically nested permutation domains. Moreover, as far as we are aware of, this is the only paper along with ours that deals with maximal domains for non-trivial Maskin monotonic rules. The key difference between our approach on maximality and the papers cited above can be (loosely) summarized as follows. The papers using restricted domains follow a "bottom-to-top" approach. A restricted possibility domain is identified and the question of its maximal enlargement is investigated. On the other hand, we follow a "top-to-bottom" approach. We start from GS/MS Theorems and study the minimal restrictions on (unrestricted) preferences domains that allow an escape from impossibility results: how much do we need to restrict the domain in order to get a possibility result. ${ }^{5}$ Our approach therefore provides a direct test of the robustness of the GS and MS Theorems. In that sense, the paper that is closest to ours is Aswal, Chatterji and Sen (2003) but their approach is antinomic to ours. While they look for ways to impose restrictions on the unrestricted domain and still preserve the impossibility result, we look for the opposite. Both papers show that one may remove exactly the same number of preference

\footnotetext{
${ }^{5}$ Closely related but different questions were posed by Kalai and Muller (1977) and Kalai and Ritz (1980). They study the general conditions for domains which admit the existence of non-dictatorial Arrow-type social welfare functions. In Kalai and Muller (1977), the domain restrictions are the same for all agents as there anonymity is one of the requirements. In Kalai and Ritz (1980), the restricted domains can be asymmetric.
} 
relation from the domain and obtain an impossibility or a possibility result. In that sense, the conclusion that the GS Theorem is far more robust than suggested by the conditions of the theorem itself should be taken with caution. Both their work and ours show that the robustness of the theorem is linked to the specific ways in which restriction operations are performed. For example our results imply that in the three alternatives case, excluding only one preference relation for only one agent yields a (maximal) domain which allows for rules that are non-dictatorial, strategy-proof and Pareto efficient. The same type of conclusion applies to the MS Theorem -although there is no paper like Aswal, Chatterji and Sen (2003) dealing with Maskin monotonicity available in the literature.

One way by which domain restrictions escape from the impossibility results is that the restriction prevents the spreading of decisiveness power. In many proofs of impossibility results decisiveness of a coalition on one pair of alternatives spreads to all pairs of alternatives. By deleting specific preferences this spreading of power is stopped. Restricting the sets of preferences for different agents may lead to very technical descriptions -see for instance Examples 7 and 8 . To the best of our knowledge there is not a complete result characterizing all those restricted domains which admit non-dictatorial, Pareto efficient and strategy-proof/Maskin monotonic rules even if we disregard the maximality of those restrictions. Finding such characterizations looks very difficult and we are not able at this stage to solve the full question -see Examples 7 and 8. We thus concentrate on a partial result and hope that this will help to solve the general case. Instead of allowing that any agent's preference domain can be restricted we restrict the preference domain of precisely one agent, say agent 1 . It will appear that in this way decisiveness power of the coalition $N \backslash\{1\}$ from which only this agent 1 is excluded is prevented to spread over all pairs. Apart from a simplification we consider this subclass interesting on its own. It answers the question what we have to know about one agent independently of the others in order to obtain a domain on which the impossibility theorems have no bite. Note that as our results are on maximal possibility domains these results provide sufficient conditions in case more than one agent preference set is restricted -see Examples 7 and 8. Clearly this is an asymmetric approach, but the non-dictatorship conditions allows for asymmetric allocation of decisiveness. A more symmetric approach at which all the admissible sets of preferences are the same intuitively seems to fit better to a case where instead of nondictatorship anonymity is invoked upon the rule. 
For strategy-proofness, we find that a domain of preferences is a maximal possibility domain if the restricted set of preferences of agent 1 has an inseparable pair or an inseparable set. In case of an inseparable pair, decisiveness of coalition $N \backslash\{1\}$ is restricted to the "reverse" of this pair where in case of an inseparable set it is restricted to any pair of alternatives belonging to this set. For Maskin monotonicity, the characterization of maximal domains is more intricate but the condition we uncover is similar to the separable set condition. ${ }^{6}$ The notion of inseparable pair is well-known in relation with non-dictatorial Arrow-type welfare functions -see e.g. Kalai and Ritz (1980). The notion of inseparable set goes at least back to Storcken (1989). Just to fix idea, let us briefly discuss the notion of inseparable pair. We say that an agent has an inseparable pair if there exist two alternatives $x$ and $y$ such that whenever $x$ is ranked best, then $y$ is second-best. The pair $(x, y)$ is then called inseparable. ${ }^{7}$ Suppose for instance that a board of managers has two vacancies. Current members of the board are contemplating several candidates. Among those are $b$, a bossy individual, and $w$ a wimp that is afraid of $b$. Then, an agent $i$ could rank $b$ at the top and $w$ second because he expects $w$ to be obedient and to copy $b^{\prime} s$ decisions-the power of $b$ would then be increased ${ }^{8}$. Clearly, agent $i$ has an inseparable pair $(b, w)$. Also a set of single peaked preferences on a finite set of alternatives possesses an inseparable pair. ${ }^{9}$

The plan of the paper is as follows. In Section 2, we introduce the model and the necessary definitions useful for the paper. In Section 3, we introduce necessary and sufficient conditions for the existence of possibility results. In Section 4 we characterize the maximal domain for non-dictatorial, Pareto efficient and strategy-proof rules. Next, in Section 5 we characterize the maximal domain for non-dictatorial, Pareto efficient and Maskin monotonic rules. Finally, we offer some concluding remarks in Section 6.

\footnotetext{
${ }^{6}$ We explain in more details this technical condition and the intuition for the proof in Section 5

${ }^{7}$ The definition of inseparable pair used by Kalai and Ritz (1980) is stronger: it says that all preferences where $x$ is preferred to $y$ these two are ranked adjacent to each other.

${ }^{8}$ On the other hand, for different preferences, this agent $i$ may like a strong candidate but dislike $b^{\prime} s$ bossiness and $w^{\prime} s$ obedience. Then, $b$ and $w$ are not necessarily ranked adjacent to each other because $i$ does not like when the power of $b$ is increased.

${ }^{9}$ Let $m$ be the number of alternatives. It is not difficult to see that the set of single peaked preferences contains $2^{(m-1)}$ preferences and the maximal set of preferences which has this inseparable pair contains $(m-1) \cdot((m-1) !)+((m-2) !)$ preferences. So, the former set of preferences is only a small fraction of the latter set of preferences.
} 


\section{The model}

There is a set of alternatives $A=\{1, \ldots, m\}$, with $m \geqslant 3$ and a set of agents $N=\{1, \ldots, n\}$ with $n \geqslant 2$. Each agent $i$ is endowed with a preference relation $p(i)$ over the alternatives of $A$ that is (strongly) complete, anti-symmetric and transitive; that is a preference relation is a linear order over alternatives. Here for a preference relation, say $p(i)$, we take the usual assumptions that $p(i)$ is a subset of $A \times A$, the two fold Carthesian product set of $A$, that for alternatives $x$ and $y$ we interpret $(x, y) \in p(i)$ as agent $i$ weakly prefers $x$ to $y$ where $(x, y) \in p(i)$ and $(y, x) \notin p(i)$ is interpreted as agent $i$ strictly prefers $x$ to $y$. Furthermore, if $B$ is a non-empty subset of alternatives then best $\left(\left.p(i)\right|_{B}\right)$ denotes the best alternative in $B$ according to agent $i$ 's preference $p(i)$, i.e. the alternative $b$ in $B$ such that for all $x$ in $B$ either $b=x$ or $b$ is strictly preferred to $x$ by agent $i$. Let $L(A)$ denote the set of all these preferences and $L(A)^{N}$ be the set of possible preference profiles. ${ }^{10}$ For different alternatives $x$ and $y, x \ldots=p(i)$ means that $x$ is the best alternative at $p(i), \ldots x \ldots y \ldots=p(i)$ means that $x$ is strictly preferred to $y$ at $p(i), \ldots x y \ldots=p(i)$ means that $x$ is strictly preferred to $y$ at $p(i)$ and there is no alternative in between these alternatives $x$ and $y$ and $x \ldots y=p(i)$ means that $x$ is the best alternative and $y$ is the worst alternative at $p(i)$. Let $L_{x}(A)$ denote the set of linear orderings that order $x$ best.

For an arbitrary relation $R$ on $A$, i.e. $R \subseteq A \times A$, the upper contour of an alternative $x$ at $R$ is defined as $u p(x, R)=\{y \in A:(y, x) \in R\}$ and the lower contour of $x$ at $R$ is defined as $\operatorname{low}(x, R)=\{y \in A:(x, y) \in R\}$. The complement of the upper contour of $x$ at $R$ is non-up $(x, R)=A-u p(x, R)$.

To model restrictions of domains of individual preferences let $\emptyset \neq L^{i} \subseteq$ $L(A)$ be the domain of individual preferences of agent $i \in N$. From now on, we assume that $L^{i}=L(A)$ if $i \geqslant 2$ and $L^{1} \varsubsetneqq L(A)$. For each alternative $a \in A$ and set of preferences $V \subseteq L(A)$, let $L_{a}^{i}=L_{a}(A) \cap L^{i}$ and $V_{a}=V \cap L_{a}(A)$. Let $A^{1}=\left\{a \in A: L_{a}^{i} \neq \emptyset\right\}$ be the set of alternatives that agent 1 can order best in at least one of his admissible preferences. Furthermore, let $I_{A^{1}}=\left\{(x, x): x \in A^{1}\right\}$ denote the identity relation on $A^{1}$. For a coalition $M$, i.e. a subset of $N$, and alternatives $x, y$ in $A$ let $L_{x}^{M} \times L_{y}^{N-M}$ denote the set of profiles $p$ such that $x \ldots=p(i)$ for all $i \in M$ and $y \ldots=p(i)$ for all

\footnotetext{
${ }^{10} L(A)^{N}$ denotes the set of all functions from $N$ to $L(A)$. We use this notation rather than the often used but equivalenet $n$-fold Carthesian product notation $L(A) \times L(A) \times$ $\ldots \times L(A)$ because the restiction of a function to a subdomain used in the definition $M$ deviation is a well-known concept and therefore needs no further explanation.
} 
$i \in N-M{ }^{11}$

A rule $K$ is a function from $L^{N}$ to $A$. For coalitions $M$ and profiles $p, q \in L^{N}$, the preference profile $p$ is said to be a $M$-deviation of a profile $q$ if $\left.p\right|_{N-M}=\left.q\right|_{N-M}$.

We now introduce the main definitions useful for the paper.

Except for intermediate strategy-proofness which can be traced back to Peters et al. (1991), the following conditions for social choice functions are well-known. We just rephrase these using the notations at hand.

Non-dictatorship: The social choice function $K$ is non-dictatorial if for each agent $i \in N$, there is a profile $p \in L^{N}$ such that $K(p) \ldots \neq p(i)$.

Pareto optimality: The social choice function $K$ is Pareto optimal if for each $(x, y) \in A^{2}$ and each $p \in L^{N}$ such that for all agents $i \in N$, $\ldots x \ldots y \ldots=p(i)$, then $K(p) \neq y$.

Strategy-proofness:The social choice function $K$ is strategy-proof if for each agent $i \in N$ and each $p, q \in L^{N}$ such that $q$ is an $\{i\}$-deviation of $p$, we have that either $K(p)=K(q)$ or $\ldots K(p) \ldots K(q) \ldots=p(i)$.

Intermediate strategy-proofness: The social choice function $K$ is $i n$ termediate strategy-proof if for each coalition $M \subseteq N$ and for each profile $p \in L^{N}$, such that there is a preference $R \in L(A)$ with $p(i)=R$ for all $i \in M$, and all $M$-deviations $q \in L^{N}$, it holds that $\ldots K(p) \ldots K(q) \ldots=R$ or $K(p)=K(q)$.

This condition means that the social choice function is not beneficial for coalitional deviations from profile $p$ to profile $q$ where at profile $p$ all members of the deviating coalition have the same preference. In Theorem 1 it is shown that intermediate strategy-proofness is equivalent to strategy-proofness on any (restricted) domain of profiles over linear orderings. It is used as a handy consequence of strategy-proofness in several proofs.

Maskin monotonicity: The social choice function $K$ is Maskin monotonic if for each $p, q \in L^{N}$,

$\operatorname{low}(K(p), p(i)) \subseteq \operatorname{low}(K(p), q(i))$ for each $i \in N$ implies that $K(p)=K(q)$.

${ }^{11}$ We set $L_{x}^{\emptyset} \times L_{y}^{N}=L_{y}^{N}$ and $L_{x}^{N} \times L_{y}^{\emptyset}=L_{x}^{N}$. 
Remark 1 We will use repeatedly in the proofs the connections between the conditions introduced above: strategy-proofness and intermediate strategyproofness are equivalent conditions, whereas the class of strategy-proof rules is (weakly) smaller than the class of Maskin monotonic rules. We provide a proof of these connections in the appendix.

Next we introduce four notions by which we can formulate the main results of this paper.

Strategy-proof possibility domain: A domain $L^{N}$ is called a strategyproof possibility domain if there exist rules $K: L^{N} \rightarrow A$ which are simultaneously non-dictatorial, Pareto efficient and strategy-proof. Furthermore, it is called a maximal strategy-proof possibility domain if it is a strategy-proof possibility domain and there is no other strategy-proof possibility domain say $\widehat{L}^{N}$, such that $L^{N} \varsubsetneqq \widehat{L}^{N}$.

Maskin monotonic possibility domain: Similarly we define a Maskin monotonic possibility domain and a maximal Maskin monotonic possibility domain by replacing the condition of strategy-proofness by the condition of Maskin monotonicity in the two previous definitions.

Decisiveness of coalitions appear also in our setting as a powerful tool to analyze the problem at hand.

Decisiveness: At rule $K$, a coalition $M \subseteq N$ is said to be decisive on $(x, y) \in A \times A$, if $K(p)=x$ for each profile $p \in L_{x}^{M} \times L_{y}^{N-M}$ and $L_{x}^{M} \times L_{y}^{N-M} \neq$ $\emptyset$.

Let $D_{K}(M)=\{(x, y) \in A \times A: M$ is decisive on $(x, y)\}$. If $K$ is Pareto efficient, then it follows immediately that $I_{A} \subseteq D_{K}(M)$. It appears that the rules which are non-dictatorial, Pareto efficient and strategyproofness/Maskin monotonic on the domains at hand are almost dictatorial. The rules by which it is proved that a domain is a possibility domain are essentially hierarchical where agent 2 is decisive on any pair in $D$ and agent 1 is decisive on the reversed remaining pairs in $A \times A^{1}$. Let $I_{A} \varsubsetneqq D \varsubsetneqq A \times A$ for some transitive relation $D$ on $A$.

Hierarchical rule: Define the hierarchical rule $K_{D}$ corresponding to $D$ as follows. For each $p \in L^{N}$

$K_{D}(p)= \begin{cases}\operatorname{best}\left(\left.p(2)\right|_{u p(y, D)}\right) & \text { for each } y \in A \text { such that } u p(y, D) \neq \emptyset \text { and } p(1) \in L_{y}^{1} \\ \operatorname{best}\left(\left.p(1)\right|_{A}\right) & \text { otherwise. }\end{cases}$ 
Now it is straightforward to see that if the pair $(y, x)$ is in $D$ then agent 2 is decisive on the pair $(x, y)$. Because of $D$ being unequal to both $I_{A}$ and $A \times A$, it follows that $K_{D}$ is non-dictatorial.

\section{Necessary and sufficient conditions for strategy- proofness and Maskin monotonicity}

We are interested in determining conditions on the domain of preferences that ensure the existence of non-dicatorial, Pareto efficient and strategyproof/Maskin monotonic rules. The necessary and sufficient conditions we uncover have two direct consequences. The first one is that we offer a possibility to directly check whether a given preference domain is respectively a strategy-proof or a Maskin monotonic possibility domain. ${ }^{12}$ The second one is that in order to escape the impossibilities stated in the GS and the MS Theorems, it is enough to destroy the product structure of the unrestricted domain by restricting the set of admissible preference relations of only one agent, say agent 1 . The specific way in which the preferences of agent 1 must be restricted will be the object of the next section where we turn our attention to the study of maximal domains.

Before proceeding to the results, we introduce in turn our central definitions for this section and we illustrate them with examples.

Strategy-proof admissibility: Let $L^{1}$ be the set of admissible preferences of agent 1 and let $D \subseteq A \times A^{1}$. The pair $\left(D, L^{1}\right)$ is strategy-proof admissible if

1. $I_{A^{1}} \varsubsetneqq D \varsubsetneqq A \times A^{1}$,

2. $D$ is transitive and

3. for each $(x, y) \in D$, each $p(1) \in L_{y}^{1}$ and each $z \in u p(x, p(1))$, both

$$
\begin{aligned}
& (z, y) \in D \text { and } \\
& (x, z) \in D \text { whenever } L_{z}^{1} \neq \emptyset .
\end{aligned}
$$

\footnotetext{
${ }^{12}$ These results are stated in Corollary 1 and Corollary 2.
} 
Let strategy $\left(D, L^{1}\right)$ stands for the strategy-proof admissibility of the pair $\left(D, L^{1}\right)$.

Before going to the definition of Maskin admissibility, it is instructive to recall the notion of a linked domain introduced in Aswal, Chatterji and Sen (2003).

Connectedness: Fix a domain of preferences $L$. A pair of alternatives $x$ and $y$ are connected, denoted $x C y$, if there exists $p(i), p^{\prime}(i) \in L$ such that $p(i)=x y \ldots$, and $p^{\prime}(i)=y x \ldots$.

Linkedness: Fix a domain of preferences $L$. Let $B \subset A$ and $x \notin B$. Then $x$ is linked to $B$ if there exists $y, z \in B$ such that $x C y$ and $x C z$.

Linked domain: The domain $L^{N}$ is a linked domain if there exists a one-to-one function $\sigma:\{1, \ldots, m\} \rightarrow\{1, \ldots, m\}$ such that

(i) $x_{\sigma(1)} C x_{\sigma(2)}$

(ii) $x_{\sigma(j)}$ is linked to $\left\{x_{\sigma(1)}, x_{\sigma(2)}, \ldots, x_{\sigma(j-1)}\right\}, j=3, \ldots, m$.

We compare next the notion of linked domain and of strategy-proof admissibility. Whereas these two notions are antinomic in nature, it is instructive to grasp some of the differences they entail on preference domains since both operate on domain restrictions.

Example 1 Let $A=\{x, y, z\}$ and let the preference domain be $L(A)$. Consider $p(i)=x y z$. Then $x C y$ since there exists $p^{\prime}(i)=y x z$. In addition, $z$ is linked to $\{x, y\}$. This implies that $L(A)$ is linked. Moreover, notice that when $m=3$ there exists no subdomain of $L(A)$ that is linked. Obviously, $L(A)^{N}$ cannot be a strategy-proof possibility domain. Suppose $(y, x) \in D$. Applying the definition of strategy-proof admissibility, we get first that $(z, x) \in D$ and $(y, z) \in D$. It is then easy to see that the decisiveness on pairs cannot be stopped so that $D=A \times A$.

Example 2 Let $A=\{x, y, z\}$ and let the preference domain be $L=\{R \in$ $L(A)$ : if $x y \ldots=R$ then $x y \ldots=R^{\prime}$ for each $\left.R^{\prime} \in L_{x}\right\}$. Given Example 1, it is clear that $L$ cannot be a linked domain. However $L^{N}$ is a strategy-proof possibility domain. To see this, let $(y, x) \in D$. Since the only preference relation $R \in L_{x}$ is $x y z=R$, there exists no alternative $z \in u p(x, R)$. Hence $D=\{(y, x)\} \cup I_{A}$. It will be made clear later that domain $L^{N}$ is not a maximal domain whereas $L^{1} \times L(A)^{N-1}$ is a maximal domain. 
Example 2 shows that, when the number of alternatives is three, we only need to remove one preference relation to recover a possibility result. We will show in the next section that this observation generalizes: given a set of alternatives of cardinality $m$, only $(m-1) !-(m-2)$ ! preference relations need to be removed to obtain a strategy-proof possibility domain. Since Aswal, Chatterji and Sen (2003) and our paper show ways to restrict domains and get, respectively, an impossibility and a possibility result, we show in the next example that both restrictions can lead to the same number of preference relations being removed. In that sense, the conclusion that the GS Theorem is far more robust than suggested by the conditions of the theorem itself should be taken with caution. In our opinion, both their work and ours show that the robustness of the theorem is linked to the specific ways in which restriction operations are performed.

Example 3 Let $A=\{w, x, y, z\}$ and let preference domains be $L=\{R \in$ $L(A)$ : there exists $x, y \in A$ such that if $x y \ldots=R$ then $x y \ldots=R^{\prime}$ for each $R^{\prime} \in$ $\left.L_{x}\right\} ; L^{\prime}=L \backslash\left\{L_{w},\{R \in L(A): \ldots . x=R\}\right\} ;$ and $L^{\prime \prime}=\{R \in L(A)$ : $x C y$ and both $w, z$ are linked to $x, y\}$. As in Example $2 L^{N}$ is a strategyproof possibility domain. For each $i \in N, L^{i}$ contains twenty orderings as opposed to 4! orderings for $L(A)$. Next it can be checked that $L^{\prime N} \subset L^{N}$ is a strategy-proof possibility domain while $L^{\prime \prime}$ is a linked domain. Notice that both $L^{\prime}$ and $L^{\prime \prime}$ contain ten orderings.

We now introduce the notion of Maskin admissibility.

Maskin admissibility: Let $L^{1}$ be the set of admissible preferences of agent 1 and let $D \subseteq A \times A^{1}$. The pair $\left(D, L^{1}\right)$ is Maskin admissible, if

1. $I_{A^{1}} \varsubsetneqq D \varsubsetneqq A \times A^{1}$,

2. $D$ is transitive and

3. for all $(x, y) \in D$ all $p(1) \in L_{y}^{1}$ all $z \in u p(x, p(1))$ and all $p(1)^{\prime} \in L_{z}^{1}$ both

$$
\begin{aligned}
& (z, y) \in D \text { and } \\
& (x, z) \in D \text { whenever } \operatorname{low}(x, p(1)) \subseteq \operatorname{low}\left(x, p(1)^{\prime}\right) .
\end{aligned}
$$

Let $\operatorname{Maskin}\left(D, L^{1}\right)$ stands for the Maskin admissibility of the pair $\left(D, L^{1}\right)$. We illustrate the notion of Maskin admissibility with several examples. 
Example 4 Let us go back to the set-up of Example 1. By the MS Theorem, strategy-proofness and Maskin monotonicity are equivalent on $L(A)$. This confirms that $D=A \times A$, thus $L(A)^{N}$ is not a Maskin monotonic possibility domain.

Example 5 Let $A$ be ordered with the binary relation $>$. The domain $L$ is single-peaked if for each $R \in L$, there exists best $(R)$ such that (i) $x>$ $y>\operatorname{best}(R)$ implies that ..y..x.. $=R$, and (ii) best $(R)>y>x$ implies that $\ldots y . . x . .=R$. For simplicity assume that $A=\{x, y, z\}$. Let $D=\{(y, x)\} \cup I_{A}$. It is easy to see that $\left(D, L^{i}\right)$ is Maskin admissible for any $i \in N$ so that $L^{N}$ is a Maskin monotonic possibility domain.

In the previous two definitions of strategy-proof admissibility and Maskin monotonic admissibility, (1) implies that $K_{D}$ is non-dictatorial, Condition (2) imposes some rationality on the decisiveness of agent 2 and together with (3) it guarantees that $K_{D}$ is strategy-proof or Maskin monotonic. Pareto efficiency follows from (3) part one.

The following Lemma shows that the condition of strategy-proof admissibility and Maskin admissibility are sufficient to guarantee that $K_{D}$ is strategy-proof or Maskin monotonic, respectively, and therewith explains the names of these two requirements on $D$ and $L^{1}$.

Lemma 1 Consider the hierarchical rule $K_{D}: L^{N} \rightarrow A$, corresponding to D. Then

1. strategy $\left(D, L^{1}\right)$ implies that $K_{D}$ is non-dictatorial, Pareto efficient and strategy-proof;

2. $\operatorname{Maskin}\left(D, L^{1}\right)$ implies that $K_{D}$ is non-dictatorial, Pareto efficient and Maskin monotonic.

Proof. Non-dictatorship follows in both cases because of $I_{A^{1}} \varsubsetneqq D \varsubsetneqq A \times A^{1}$. To prove Pareto efficiency let $p$ be a profile with $p(1) \in L_{y}^{1}$. It is sufficient to prove that Pareto efficiency is satisfied at this profile $p$. This is indeed the case whenever $K_{D}(p)=y$. So, suppose that $K_{D}(p)=\operatorname{best}\left(\left.p(2)\right|_{u p(y, D)}\right)$ and $K_{D}(p) \neq y$. Because of $\operatorname{strategy}\left(D, L^{1}\right)$ as well as $\operatorname{Maskin}\left(D, L^{1}\right)$, it follows that $z \in u p(y, D)$ if there are $x \in u p(y, D)$ and $R \in L_{y}^{1}$ with $y \ldots z \ldots x \ldots=R$. So, for all $a \in$ non-up $(y, D)$, we have $\ldots K_{D}(p) \ldots a \ldots=p(1)$ and for all $a \in$ 
$u p(y, D)-\left\{K_{D}(p)\right\}$, we have $\ldots K_{D}(p) \ldots a \ldots=p(2)$. So, Pareto efficiency is satisfied at $p$.

In order to prove (1), assume strategy $\left(D, L^{1}\right)$. Furthermore, let $p$ be as above and profile $q$ an $\{i\}$-deviation of $p$ for some agent $i$ with $q(1) \in L_{z}^{1}$ for some $z \in A$. To the contrary suppose that $\ldots K_{D}(q) \ldots K_{D}(p) \ldots=p(i)$. By the definition of $K_{D}$ it follows that $i \leqslant 2$. If $i=2$, then $p(1)=q(1)$. Consequently, $y=z$ and $K_{D}(q)=\operatorname{best}\left(\left.q(2)\right|_{u p(z, D)}\right)=\operatorname{best}\left(\left.q(2)\right|_{u p(y, D)}\right)$. So, in that case $K_{D}(q) \in u p(y, D)$. Because $K_{D}(p)=\operatorname{best}\left(\left.p(2)\right|_{u p(y, D)}\right)$, we obtain a contradiction with $\ldots K_{D}(q) \ldots K_{D}(p) \ldots=p(i)$. To conclude the proof of $(1)$, let $i=1$. Therefore, $p(2)=q(2)$. Because of $\ldots K_{D}(q) \ldots K_{D}(p) \ldots=p(1)$ and $\operatorname{strategy}\left(D, L^{1}\right)$, it follows that $K_{D}(p) \in u p\left(K_{D}(q), D\right)$. Thus, since $D$ is transitive, we conclude that $K_{D}(p) \in u p(z, D)$. But then $K_{D}(q)=$ $\operatorname{best}\left(\left.q(2)\right|_{u p(z, D)}\right)=\operatorname{best}\left(\left.p(2)\right|_{u p(z, D)}\right)$ and $\ldots K_{D}(q) \ldots K_{D}(p) \ldots=p(1)$ contradict each other. This proves strategy-proofness.

In order to prove (2), assume $\operatorname{Maskin}\left(D, L^{1}\right)$ and consider $\{i\}$-deviations $p$ and $q$ as before with, in addition, $\operatorname{low}\left(K_{D}(p), p(i)\right) \subseteq \operatorname{low}\left(K_{D}(p), q(i)\right)$. It is sufficient to prove that $K_{D}(p)=K_{D}(q)$. This obviously holds whenever $i \geqslant 2$. So suppose that $i=1$. Since $\operatorname{low}\left(K_{D}(p), p(1)\right) \subseteq \operatorname{low}\left(K_{D}(p), q(1)\right)$, it follows that $z \in u p\left(K_{D}(p), p(1)\right)$. Maskin $\left(D, L^{1}\right)$, or reflexivity of $D$, or $z=y$ imply that $\left(K_{D}(p), z\right) \in D$ and $(z, y) \in D$. Furthermore, transitivity of $D$ implies that $u p(z, D) \subseteq u p(y, D)$. But because $K_{D}(p)=\operatorname{best}\left(\left.p(2)\right|_{u p(y, D)}\right)$, it follows that $K_{D}(p)=\operatorname{best}\left(\left.p(2)\right|_{u p(y, D)}\right)=\operatorname{best}\left(\left.p(2)\right|_{u p(z, D)}\right)=\operatorname{best}\left(\left.q(2)\right|_{u p(z, D)}\right)=$ $K_{D}(q)$.

We now prove that the conditions of strategy-proof admissibility and Maskin admissibility are in fact also necessary to guarantee the existence of rules that are non-dictatorial, Pareto efficient and strategy-proof/Maskin monotonic. For the rest of this section, let $K$ be a non-dictatorial and Pareto efficient. We will show that whenever $K$ is strategy-proof or Maskin monotonic, then $I_{A^{1}} \varsubsetneqq D_{K}(N-\{1\}) \varsubsetneqq A \times A^{1}$ is transitive and that the pair $\left(D_{K}(N-\{1\}), L^{1}\right)$ is respectively strategy-proof admissible or Maskin monotonic admissible.

To avoid needless repetitions, assume that $K$ is at least Maskin monotonic. So, cases at which $K$ is strategy-proof are spelled out explicitly. The following Lemma formulates a condition when decisiveness at a specific profile spreads to decisiveness on a specific pair. Actually this condition coincides with the linking condition of Aswal, Chatterji and Sen (2003) introduced at the beginning of the section. 
Lemma 2 Let $p \in L^{N}, M \subseteq N$ and $(x, y) \in A \times(A \backslash\{x\})$ such that for each agent $i \in M, x y \ldots=p(i)$ and for each agent $i \in N-M, y \ldots=p(i)$. Let $K(p)=x$. Then $(x, y) \in D_{K}(M)$.

Proof. By the assumptions on $p$ we have that $L_{x}^{M} \times L_{y}^{N-M} \neq \emptyset$. Let $q \in L_{x}^{M} \times L_{y}^{N-M}$. It is sufficient to prove that $K(q)=x$. Let $r \in L^{N}$ be an $(N-M)$-deviation of $p$ and an $M$-deviation of $q$. Now, Pareto efficiency implies that $K(r) \in\{x, y\}$. If $K(r)=y$, then Maskin monotonicity would imply the contradiction that $K(p)=y$. Therefore, $K(r)=x$. But then Maskin monotonicity implies that $K(q)=x$.

Lemma $3 I_{A^{1}} \varsubsetneqq D_{K}(N-\{1\}) \varsubsetneqq A \times A^{1}$ and $D_{K}(N-\{1\})$ is transitive.

Proof. (Proof of $I_{A^{1}} \varsubsetneqq D_{K}(N-\{1\})$ ) To the contrary, suppose that $I_{A^{1}}=$ $D_{K}(N-\{1\})$. We show that this leads to the contradiction that agent 1 is a dictator. Consider $R \in L^{1}$ and a profile $p \in L^{N}$, with $x y \ldots=R=p(1)$ for some $x$ and $y$. To deduce the contradiction, it is sufficient to prove that $K(p)=x$. Consider the profiles $q$ and $r$-both $N-\{1\}$-deviations of $p$ such that $q(i)=y x \ldots$ and $r(i)=y \ldots x$ for each $i \geqslant 2$. Because of Pareto efficiency, it follows that $K(q) \in\{x, y\}$. Now, by Lemma 2, it follows that $K(q) \neq y$, otherwise $(y, x) \in\left(D_{K}(N-\{1\})-I_{A^{1}}\right)$. But then $K(q)=x$ and $(x, y) \in D_{K}(\{1\})$. So, $K(r)=x$. By Maskin monotonicity, we obtain that $K(p)=x$.

(Proof of $\left.D_{K}(N-\{1\}) \varsubsetneqq A \times A^{1}\right)$ To the contrary suppose that $D_{K}(N-$ $\{1\})=A \times A^{1}$. We show that this leads to the contradiction that $K$ is dictatorial. For $R \in L^{1}$, consider the rule $K^{R}$ defined by $K^{R}(p)=K(R, p)$ for each profile $p \in L(A)^{N-\{1\}}$. It is clear that $K^{R}$ is surjective-and even unanimous-and Maskin monotonic. By Lemma 1, it is strategy-proof. Hence, by the GS Theorem, it follows that $K^{R}(p)$ is dictatorial, say by agent $i_{R} \geqslant 2$.

Consider two preferences $R$ and $R^{\prime}$ in $L^{1}$. In order to prove that $K$ is dictatorial, it is sufficient to show that $i_{R}=i_{R^{\prime}}$. To the contrary, suppose that $i_{R} \neq i_{R^{\prime}}$. We deduce a contradiction and are done. Obviously there are different alternatives $x, y, z_{1}, z_{2}, \ldots z_{k-1}$ and $z_{k}$, where $k$ may be zero, such that $z_{1} z_{2} \ldots z_{k} x \ldots=R$ and $z_{1} z_{2} \ldots z_{k} y \ldots=R^{\prime}$. So $x$ and $y$ are the first alternatives on which the preferences $R$ and $R^{\prime}$ differ. Consider profiles $p$ and $q$ which are $\{1\}$-deviations such that $p(1)=R, q(1)=R^{\prime}, p\left(i_{R}\right)=q\left(i_{R}\right)=y \ldots$ and $p\left(i_{R^{\prime}}\right)=q\left(i_{R^{\prime}}\right)=x \ldots$. Then since $i_{R}$ is a dictator at $K^{R}$, it follows that $K(p)=y$ and because $i_{R^{\prime}}$ is a dictator at $K^{R^{\prime}}$, it follows that $K(q)=x$. 
Finally, because $l o w(y, R) \subseteq \operatorname{low}\left(y, R^{\prime}\right)$, we have a contradiction with Maskin monotonicity of $K$.

(Proof of transitivity) Let $(x, y),(y, z) \in D_{K}(N-\{1\})$. It is sufficient to prove that $(x, z) \in D_{K}(N-\{1\})$. This is trivially the case when $x, y$ and $z$ are not three different alternatives. So, let these three alternatives be different. Let $R=z \ldots \in L^{1}$ arbitrary. Consider $p \in L^{N}$ such that $p(1)=R$ and $p(i)=x z \ldots$ for each $i \in N-\{1\}$. By Lemma 2, it is sufficient to prove that $K(p)=x$. Consider $(N-\{1\})$-deviation $q$ of $p$ such that $x y z \ldots=q(i)$ for all $i \in N-\{1\}$. If $K(q)=y$, then Maskin monotonicity would yield $K(r)=y$-where $r$ is the $\{1\}$-deviation of $q$ with $r(1)=y \ldots-$ which contradicts $(x, y) \in D_{K}(N-\{1\})$. If $K(q)=z$, then Maskin monotonicity would yield $K(u)=z$, where $u$ is a $(N-\{1\})$-deviation of $q$, with $y z \ldots=u(i)$ for all $i \in N-\{1\}$, which contradicts $(y, z) \in D_{K}(N-\{1\})$. Therefore $K(q)=x$ and Maskin monotonicity implies that $K(p)=x$.

Lemma 4 Let $x, y$ and $z$ be different alternatives and $R \in L^{1}$ such that $y \ldots z \ldots \ldots \ldots=R$. Let $(x, y) \in D_{K}(N-\{1\})$. Then

1. $(z, y) \in D_{K}(N-\{1\})$;

2. $(x, z) \in D_{K}(N-\{1\})$ if $K$ is strategy-proof and $L_{z}^{1} \neq \emptyset$;

3. $(x, z) \in D_{K}(N-\{1\})$ if there are $R^{\prime} \in L_{z}^{1}$ such that low $(x, R) \subseteq$ low $\left(x, R^{\prime}\right)$.

Proof. (Proof of 1) Let $q \in L^{N}$ such that $q(1)=R$ and $z y \ldots=q(i)$ for all $i \in N-\{1\}$. By Lemma 2 it is sufficient to prove that $K(q)=z$. Consider $p$ and $r$, two $(N-\{1\})$-deviations of $q$, such that $x z y \ldots=p(i)$ and $z x y \ldots=r(i)$ for all $i \in N-\{1\}$. Because of $(x, y) \in D(N-\{1\})$, it follows that $K(p)=x$. Pareto efficiency implies that $K(r) \in\{y, z\}$. If $K(r)=y$, then Maskin monotonicity would yield the contradiction that $K(p)=y$. Therefore, $K(r)=z$ and Maskin monotonicity implies that $K(q)=z$.

(Proof of 2) Suppose $K$ is strategy-proof and $z \ldots=R^{\prime} \in L_{z}^{1} \neq \emptyset$. Let $q \in L^{N}$ such that $q(1)=R^{\prime}$ and $x z \ldots=q(i)$ for all $i \in N-\{1\}$. By Lemma 2 , it is sufficient to prove that $K(q)=x$. Consider $(N-\{1\})$-deviations $p$ of $q$, with $p(1)=R$. Because of $(x, y) \in D(N-\{1\})$, it follows that $K(p)=x$. Pareto efficiency implies that $K(q) \in\{x, z\}$. Now considering $p$ and $q$, it follows that $K(q)=z$ would violate strategy-proofness. In consequence, $K(q)=x$. 
(Proof of 3) Suppose $R^{\prime} \in L_{z}^{1}$ such that $\operatorname{low}(x, R) \subseteq \operatorname{low}\left(x, R^{\prime}\right)$. Let $q \in L^{N}$ be such that $q(1)=R^{\prime}$ and $x z \ldots=q(i)$ for all $i \in N-\{1\}$. By Lemma 2 , it is sufficient to prove that $K(q)=x$. Consider $(N-\{1\})$-deviations $p$ of $q$, with $p(1)=R$. Because of $(x, y) \in D(N-\{1\})$, it follows that $K(p)=x$. Next, because $\operatorname{low}(x, R) \subseteq \operatorname{low}\left(x, R^{\prime}\right)$ and Maskin monotonicity, we have that $K(q)=x$.

Combining Lemma's 1, 2, 3 and 4 yields the following corollaries which are the central results of this section.

Corollary $1 L^{N}$ is a strategy-proof possibility domain if and only if there exists $i \in N$ such that $\left(D_{K}(N-\{i\}), L^{i}\right)$ is strategy-proof admissible.

Corollary $2 L^{N}$ is a Maskin monotonic possibility domain if and only if there exists $i \in N$ such that $\left(D_{K}(N-\{i\}), L^{i}\right)$ is Maskin admissible;

\section{Maximal domains for strategy-proofness}

In the preceeding section, we introduced ways to check whether a given domain is respectively a strategy-proof or a Maskin monotonic possibility domain. We now study the specific way to make a domain a strategy-proof possibility domain. Moreover, we look for domain restrictions that are minimal. That is the preference domain obtained is a maximal domain: any enlargement would make the domain to be dictatorial. As emphasized in the introduction, our approach can thus be seen as the opposite of Aswal, Chatterji and Sen (2003). Since we already know from the previous section that the preferences of only one agent need to be restricted in order to obtain a possibility domain, we assume that $L^{1} \subset L(A)$ while $L^{i}=L(A)$ for each $i \neq 1$.

Maximal strategy-proof possibility domains are characterized by the following two inseparability notions.

Inseparable pair: The set of preferences $L^{i}$ has an inseparable pair $(x, y) \in A \times(A \backslash\{x\})$ if $x y \ldots=R$ for each $R \in L_{x}^{i}$.

So, the ordered pair of alternatives $(x, y)$ is an inseparable pair of the set of admissible preferences of agent $i$ if $y$ is ordered second best in all preferences where $x$ is ordered best. It is already mentioned in the introduction that on a finite set of alternatives the set of single peaked preferences has an inseparable 
pair. To be more specific on this let the set of alternatives be $\left\{a_{1}, a_{2}, \ldots, a_{m}\right\}$. Consider all single peaked preferences with respect to the basic order $>$ with $a_{1}>a_{2}>\ldots>a_{m}$. That is at such a preference say $R$ there is an alternative say $a_{t}$ such that $a_{t} \ldots a_{t+1} \ldots a_{m} \ldots=R$ and $a_{t} \ldots a_{t-1} \ldots a_{2} \ldots a_{1} \ldots=R$. Now both $\left(a_{1}, a_{2}\right)$ and $\left(a_{m}, a_{m-1}\right)$ are inseparable pairs. An other example of set of preferences with an inseparable pair is as follows. Let there be precisely three alternatives say $a, b$ and $c$. Let $L^{1}=L(\{a, b, c\})-\{R\}$, where $a b c=R$. Then $L^{1}$ has inseparable pair $(a, c)$.

Inseparable set: Let $B$ be a subset of $A$, with $3 \leqslant \# B<m$. The set of preferences $L^{i}$ has an inseparable set $B$, with $3 \leqslant \# B<m$, if for all $b \in B$, all $R \in L_{b}^{i}$, all $a \in B$ and all $c \in A-B$, we have ...a...c... $=R$.

So, for all preferences $R$ in $L^{i}$, if the best alternative at $R$ is in $B$, then $B$ is preferred to $(A-B)$ at $R$. Note that this condition trivially holds for the empty set, any singleton set and the set $A$ itself. Therefore these are excluded. Furthermore, if $B$ consists of precisely two alternatives, then having an inseparable set means having two inseparable pairs, which explains why sets with cardinality 2 are excluded in the definition of inseparable set. 13

Corollary 1 characterizes strategy-proof possibility domains in terms of a set of pairs of alternatives on which the coalition of agents whose sets of preferences are not restricted are decisive. Consider a non-dictatorial, Pareto efficient and strategy-proof rule $K$, such that for all strategy-proof possibility domains $\widehat{L}^{N}$ with $L^{N} \subseteq \widehat{L}^{N}$, we have that $L^{N}=\widehat{L}^{N}$. So, $L^{N}$ is a maximal strategy-proof possibility domain. We shall prove in Theorem 1 that the conditions on $D_{K}(N-\{1\})$ under this maximality property yield that $L^{1}$ either has one inseparable pair or one inseparable set. Furthermore, we deduce that these separabilities are not only necessary but also sufficient. The following example shows why these inseparabilities imply that a domain at hand is a strategy-proof possibility domain.

\footnotetext{
${ }^{13}$ The notion of inseparable pair used in Kalai and Ritz(1980) states that $L^{i}$ has an inseparable pair $(x, y)$ if for all $R \in L^{i}$ if $\ldots x \ldots y \ldots=R$, then $\ldots x y \ldots=R$. Here we only need this inseparability if $x$ is top alternative, because the almost dictatorial choice function depends mainly on the top alternatives of agent 1 , the agent with the restricted preference set. In Aswal, Chatterji and Sen (2003) it is called the unique seconds property, to emphasize its origin we stick to the name as used here. A similar remark as for inseparable pair holds for inseparable set as defined in Storcken (1989).
} 
Example 6 In case $L^{1}$ has an inseparable pair $(y, x)$ or an inseparable set $B$, it follows straightforwardly that strategy $\left(D, L^{1}\right)$, where $D=\{(x, y)\} \cup$ $I_{A}$ or $D=(B \times B) \cup I_{A}$ respectively. So, Lemma 1 implies that in these situations, the hierarchical rule $K_{D}$ is non-dictatorial, Pareto efficient and strategy-proof. Thus, in that case, $L^{N}$ is a strategy-proof possibility domain. In the case of an inseparable pair $(y, x)$ and with $D=\{(x, y)\} \cup I_{A}, K_{D}$ then simplifies to: For each $p \in L^{N}$

$$
K_{D}(p)= \begin{cases}\operatorname{best}\left(\left.p(2)\right|_{x, y)}\right) & \text { if } p(1) \in L_{y}^{1} \\ \operatorname{best}\left(\left.p(1)\right|_{A}\right) & \text { otherwise. }\end{cases}
$$

The following Lemmas are needed for the proof of our first Theorem.

Lemma 5 Let $x \in A$. Then $L_{x}^{1} \neq \emptyset$.

Proof. To the contrary let $L_{x}^{1}=\emptyset$. Take $\widehat{L}^{1}=L^{1} \cup\{R \in L(A): x y \ldots=R\}$ for some fixed $y \in A-\{x\}$. Clearly, by taking $\widehat{L}^{i}=L(A)$ for $i>1$, we obtain that $L^{N} \varsubsetneqq \widehat{L}^{N}$. By lemma 1, the latter is a strategy-proof possibility domain. This yields a contradiction with $L^{N}$ being maximal.

Intuitively it is reasonable that the smaller the set of decisive pairs of $N-\{1\}$, the larger $L^{1}$ can be taken. The following Lemma shows that we may shrink the set of decisive pairs of $N-\{1\}$.

Lemma 6 Let $\left(x^{*}, y^{*}\right) \in D_{K}(N-\{1\})$, with $x^{*} \neq y^{*}$.

Take

$$
\begin{gathered}
Y=\left\{a \in A: a=y^{*} \operatorname{or}\left(a, y^{*}\right),\left(y^{*}, a\right) \in D_{K}(N-\{1\})\right\} \text { and }, \\
Z=\left\{a \in A:\left(a, y^{*}\right) \in D_{K}(N-\{1\}) \operatorname{and}\left(y^{*}, a\right) \notin D_{K}(N-\{1\})\right\} .
\end{gathered}
$$

Let $D^{*}=\left[(Z \times Z) \cup(Z \times Y) \cup(Y \times Y) \cup I_{A}\right] \cap D_{K}(N-\{1\})$.

Then strategy $\left(D^{*}, L^{1}\right)$. Furthermore, $\left[(Z \times Y) \cup(Y \times Y) \cup I_{A}\right] \subseteq D_{K}(N-$ $\{1\})$.

Proof. By definition $\left(x^{*}, y^{*}\right) \in D^{*}-I_{A}$ and $D^{*} \subseteq D_{K}(N-\{1\})$. So, $I_{A} \varsubsetneqq D^{*} \varsubsetneqq A \times A$. Because of transitivity of $D_{K}(N-\{1\})$ and $[(Z \times Z) \cup$ $\left.(Z \times Y) \cup(Y \times Y) \cup I_{A}\right]$ it follows that $D^{*}$ is transitive. Let $x, y, z \in A$, with $\#\{x, y, z\}=3$. Let $(x, y) \in D^{*}$. Let $y \ldots z \ldots x \ldots=R$ in $L^{1}$. It is sufficient to 
prove that $(z, y) \in D^{*}$ and $(x, z) \in D^{*}$. Because of strategy $\left(D_{K}(N-\{1\}), L^{1}\right)$ and Lemma 5 , it follows that $(x, z),(z, y) \in D_{K}(N-\{1\})$. Moreover, by transitivity of $D_{K}(N-\{1\})$ and the definition of $Y$ and $Z$, it follows that $D_{K}(N-\{1\}) \cap(Y \times Z)=\emptyset$. Thus, it is sufficient to prove that $z \in Y \cup Z$. Because of $y \in Y \cup Z$, it follows that $\left(y, y^{*}\right) \in D_{K}(N-\{1\})$. However, $(z, y) \in D_{K}(N-\{1\})$ and transitivity of $D_{K}(N-\{1\})$ together imply that $\left(z, y^{*}\right) \in D_{K}(N-\{1\})$. Therefore, $z \in Y \cup Z$.

The furthermore part follows by transitivity of $D_{K}(N-\{1\})$ and the definition of $Y$ and $Z$.

Lemma 7 There are disjoint subsets $Y$ and $Z$ of $A$ such that

1. $Y$ is non-empty, $Y \cup Z \neq A$, and $\#(Y \cup Z) \geqslant 2$;

2. $I_{A} \varsubsetneqq\left[((Y \cup Z) \times Y) \cup I_{A}\right] \subseteq D_{K}(N-\{1\}) \varsubsetneqq A \times A$ and

3. $\operatorname{strategy}\left(((Y \cup Z) \times Y) \cup I_{A}, L^{1}\right)$.

Proof. In Lemma 6, we proved that there are disjoint $Y$ and $Z$ such that strategy $\left(\left[(Z \times Z) \cup(Z \times Y) \cup(Y \times Y) \cup I_{A}\right] \cap D_{K}(N-\{1\}), L^{1}\right)$. Because of the transitivity of $D_{K}(N-\{1\})$, we may take $\left(x^{*}, y^{*}\right) \in D_{K}(N-\{1\})$ such that $x^{*} \neq y^{*}$ and $(Z \times Z) \cap D_{K}(N-\{1\}) \subseteq I_{A}$. By the furthermore part of the previous Lemma 6 , we obtain that $(Y \cup Z) \times Y \subseteq D_{K}(N-\{1\})$. Hence, it follows that strategy $\left(((Y \cup Z) \times Y) \cup I_{A}, L^{1}\right)$. The inclusions at (2) follow readily.

Also, by definition, $Y$ is non-empty and because of $x^{*}, y^{*} \in Y \cup Z$, with $x^{*} \neq y^{*}$, we have $\#(Y \cup Z) \geqslant 2$.

It remains to prove that $Y \cup Z \neq A$. In order to do so, suppose that $Y \cup Z=A$. We prove that we may take $Z=\emptyset$ and that $Y \neq A$. Consider $y \in Y$ and $R=y \ldots a$ in $L^{1}$ where $a \in A$. Because of strategy $(((Y \cup Z) \times Y) \cup$ $\left.I_{A}, L^{1}\right)$ and $(a, y) \in((Y \cup Z) \times Y)$, it follows for each $b \in A-\{y, a\}$ that $(a, b) \in((Y \cup Z) \times Y)$. Hence, $b \in Y$ for each $b \in A-\{a, y\}$. Next, because $\left[((Y \cup Z) \times Y) \cup I_{A}\right] \varsubsetneqq A \times A$, it follows that $Z \neq \emptyset$. But then $Z=\{a\}$. As the previous holds for every $a^{\prime} \in A$ for which there are $y \in Y, R \in L_{y}^{1}$ with $y \ldots a^{\prime}=R$ and $Z$ is a singleton, it follows that for each $y \in Y$ and each $R \in L_{y}^{1}$ that $y \ldots a=R$. Hence, strategy $\left((Y \times Y) \cup I_{A}, L^{1}\right)$ and as $Z$ is a singleton $Y \neq A$. By taking $Z=\emptyset$ this shows the existence of such $Y$ and $Z$. 
Theorem 1 Let $L^{N}$ be such that $L^{i}=L(A)$ for all $i \geqslant 2$. Then $L^{N}$ is a maximal strategy-proof possibility domain if and only if one of the following two conditions holds

1. There are $a, b \in A$ with $a \neq b$ and $L^{1}=V$ where $V=\{R \in L(A)$ : if $R \in L_{a}^{1}$, then $\left.a b \ldots=R\right\}$ which means that $L^{1}$ has an inseparable pair $(a, b)$;

2. There is a non-trivial subset $Y$ of $A$, with $\# Y \geqslant 3$, such that $L^{1}=W$ where $W=\left\{R \in L(A)\right.$ : if $R \in L_{y}^{1}$ for some $y \in Y$, then for all $a \in Y$ and all $b \in A-Y$ we have ...a...b... $=R\}$ which means that $L^{1}$ has an inseparable set $Y$.

Proof. (Only-if-part) Suppose $L^{1}$ is a maximal strategy-proof possibility domain. By the previous Lemma there are disjoint subsets $Y$ and $Z$ of $A$ such that

1. $Y$ is non-empty, $Y \cup Z \neq A$, and $\#(Y \cup Z) \geqslant 2$;

2. $I_{A} \varsubsetneqq\left[((Y \cup Z) \times Y) \cup I_{A}\right] \varsubsetneqq A \times A$ and

3. strategy $\left(((Y \cup Z) \times Y) \cup I_{A}, L^{1}\right)$.

Consider $y \in Y$ and $z, t \in Y \cup Z$. Take $R \in L_{y}^{1}$ which exist by Lemma 5 and assume without loss of generality that $y \ldots t \ldots z \ldots=R$. Then by strat$\operatorname{egy}\left(((Y \cup Z) \times Y) \cup I_{A}, L^{1}\right)$, it follows that $(z, t) \in(Y \cup Z) \times Y$. Hence, $t \in Y$. So, $\# Z \leqslant 1$. Moreover, if $z \in Z$, then for all $t \in Y-\{y\}$, we have $y \ldots t \ldots z \ldots=R$. Because $y$ is chosen arbitrarily, this means that strat$\operatorname{egy}\left((Y \times Y) \cup I_{A}, L^{1}\right)$ or $\# Y=\# Z=1$. Suppose $Y=\{b\}$ and $Z=\{a\}$. Then strategy $\left(((Y \cup Z) \times Y) \cup I_{A}, L^{1}\right)$ implies that $L^{1}$ has an inseparable pair $(a, b)$. Consider the set $V$. We shall prove $L^{1}=V$. Because obviously strat$\operatorname{egy}\left(((Y \cup Z) \times Y) \cup I_{A}, V\right)$ and therefore strategy $\left(((Y \cup Z) \times Y) \cup I_{A}, L^{1} \cup V\right)$ it follows by the maximality of $L^{1}$ that $V \subseteq L^{1}$. Now $V$ is defined such that it contains all sets of preferences which have an inseparable pair $(a, b)$. Therefore $L^{1} \subseteq V$. So, $L^{1}=V$. Now suppose strategy $\left((Y \times Y) \cup I_{A}, L^{1}\right)$. Then it follows that $L^{1}$ has an inseparable set $Y$. Consider the set $W$. By proving that $L^{1}=W$, we end the proof of the only-if-part. Because obviously strat$\operatorname{egy}\left((Y \times Y) \cup I_{A}, W\right)$ and therefore obviously strategy $\left((Y \times Y) \cup I_{A}, L^{1} \cup W\right)$, it follows by the maximality of $L^{1}$ that $W \subseteq L^{1}$. But $W$ is defined such that 
is contains all sets of preferences which have an inseparable set $Y$. Therefore $L^{1} \subseteq V$. So, $L^{1}=W$.

(If-part) By example 6 it is clear that if $L^{1}$ equals either $V$ or $W$, then $L^{N}$ is a strategy-proof possibility domain. It remains to prove the maximality of it. Suppose $L^{i} \subseteq \widehat{L}^{i}$ for all agents $i$ and $\widehat{L}^{N}$ is a maximal strategy-proof possibility domain. It is sufficient to prove that $L^{N}=\widehat{L}^{N}$. By the only-ifpart, it follows that $\widehat{L}^{1}$ has either an inseparable set say $\widehat{Y}$ or an inseparable pair say $(\widehat{a}, \widehat{b})$. Because $L^{1} \subseteq \widehat{L}^{1}$ and is such that it contains all sets of preferences which either have an inseparable set $Y$ or an inseparable pair $(a, b)$, it follows that the inseparable sets or pairs are equal and that $L^{1}=\widehat{L}^{1}$.

Remark 2 In Theorem 1 we used hierarchical rules to show that a domain with an inseparable pair or set is a strategy-proof possibility domain. In fact any rule $K$ such that $D_{K}(N-\{1\})$ is either equal to $\{(x, y)\} \cup I_{A}$ or equal to $(B \times B) \cup I_{A}$ for some different alternatives $x$ and $y$ or for a non-trivial subset $B$ of $A$ with at least three alternatives can be used here. For instance in case $D_{K}(N-\{1\})=\{(x, y)\} \cup I_{A}$ one might define $K$ non-hierarchically as follows. For all profiles $p$ in $L^{N}$

$$
K_{D}(p)= \begin{cases}x & \text { if best }\left(\left.p(1)\right|_{A}\right)=y \text { and } \ldots x \ldots y \ldots=p(i) \text { for some } i \neq 1 \\ z & \text { in all other cases where } z=\operatorname{best}\left(\left.p(1)\right|_{A}\right) .\end{cases}
$$

\section{Maximal domains for Maskin monotonicity}

In this section we characterize the maximal Maskin monotonic possibility domains for the case that precisely one agent's set of preferences is restricted. Our next Theorem spells out a characterization of Maskin monotonic possibility domains in terms of a set of pairs of alternatives on which the coalition of agents whom sets of preferences are not restricted are decisive.

Not surprisingly, the characterization of maximal domains is more intricate than in the strategy-proof case. It relies on the existence of disjoint subsets $Y$ and $Z$ of the sets of alternative and the existence of an asymmetric and transitive relation $P$ on the set of alternatives. Now coalition $N-\{1\}$ is decisive on all pairs in $Y$ and all pairs $(z, y)$ such that $z$ is in $Z$ and $y$ is in $Y$. To achieve that this decisiveness does not spread further it is on the one hand required that at all admissible preferences for agent 1, with the best alternative in $Y$, all alternatives in subset $Y \cup Z$ are preferred to all those 
not in this union. Actually this requirement follows from condition (a) of Theorem 2 and part 1 of condition (d) of this Theorem introduced below. ${ }^{14}$ This condition is similar to the separable set condition. As this is the only requirement on the set $Y$ it is not necessarily "separated" from $Z$. That is there may be alternatives $z$ in $Z$ which are preferred to an alternative in $Y$ at a preference with its best element in $Y$. Therefore on the other hand it is required that decisiveness on a pair $(z, y)$ or $\left(y^{\prime}, y\right)$ cannot spread to a pair $\left(z, z^{\prime}\right)$ or to a pair $\left(y, z^{\prime}\right)$ for alternatives $z$ and $z^{\prime}$ in $Z$ and $y$ and $y^{\prime}$ in $Y$. Now part 2 of condition (d) in Theorem 2 essentially takes care that, in these situations, Maskin monotonicity has no bite.

Remark 3 Consider Lemmas 5', 6' and 7' obtained from Lemmas 5, 6 and 7 by replacing the word "strategy" by the word "Maskin" respectively. The proofs of these Lemma's follow likewise by the same substitution in the proofs of the original Lemma's. To avoid obvious repetitions neither the Lemma's 5', 6' and ' '' nor their proofs are written out here.

Theorem 2 Let $L^{N}$ be such that $L^{i}=L(A)$ for all $i \geqslant 2$. Then $L^{N}$ is a maximal Maskin monotonic possibility domain if, and only if, there are disjoint subsets $Y$ and $Z$ of $A$ and an asymmetric and transitive relation $P$ on A such that

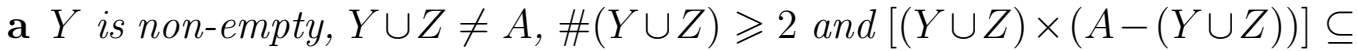
$P \subseteq[(Y \cup Z) \times(A-Y)]$

$\mathbf{b}$ if $L^{1}$ has a separable pair $(b, a)$ then $Y=\{b\}$ and $Z=\{a\}$;

c there is no partition $X_{1}, X_{2}$ of $Y \cup Z$ with $\# X_{1} \geqslant 2$ and $X_{1} \times X_{2} \subseteq P$ and

d $L^{1}=V$ where $V=\{R \in L(A)$ :

1. if $y \ldots=R$ for some $y \in Y$, then $P \subseteq R$;

2. if $z \ldots=R$ for some $z \in Z$, then there are $t \in A-(Y \cup Z)$ such that $[($ non-up $(z, P)-\{z\}) \cap(Y \cup Z)] \subseteq \operatorname{low}(t, R)\}$.

\footnotetext{
${ }^{14}$ These two yield $(Y \cup Z) \times Y \subseteq P \subseteq R$, where $R$ is such an admissible preference of agent 1 with best element in $Y$.
} 
Proof. (Only-if-part) Let $L^{N}$ be a maximal Maskin monotonic possibility domain. We will show the existence of sets $Y, Z$ and relation $P$ satisfying the conditions formulated in the theorem. By Lemma 7' we have $Y, Z \subseteq A$,

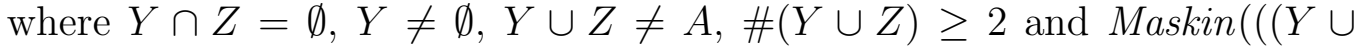
$\left.Z) \times Y) \cup I_{A}, L^{1}\right)$. For different alternatives $a$ and $b$, define $(a, b) \in P$ if for all $R \in L_{y}^{1}$ and all $y \in Y$ we have that ...a...b. $=R$. By definition, $P$ is asymmetric and because the preferences in $L^{1}$ are transitive, $P$ is transitive. Because of Maskin $\left(((Y \cup Z) \times Y) \cup I_{A}, L^{1}\right)$, it follows that $[(Y \cup Z) \times(A-$ $(Y \cup Z))] \subseteq P$. Because of the definition of $P$ and Lemma 5 it follows that $P \subseteq[(Y \cup Z) \times(A-Y)]$. This proves part (a).

Claim 1 Maskin $\left(((Y \cup Z) \times Y) \cup I_{A}, V\right)$.

We have to prove the following implications for $x, y, z \in A$ and $R, R^{\prime} \in V$.

1. If $(x, y) \in(Y \cup Z) \times Y$ and $y \ldots z \ldots x \ldots=R$, then $(z, y) \in(Y \cup Z) \times Y$;

2. If $(x, y) \in(Y \cup Z) \times Y, y \ldots z \ldots x \ldots=R, z \ldots=R^{\prime}$ and $\operatorname{low}(x, R) \subseteq$ low $\left(x, R^{\prime}\right)$, then $(x, z) \in(Y \cup Z) \times Y$.

The proof of the first implication follows immediately from the first condition in the definition of $V$ and $[(Y \cup Z) \times(A-(Y \cup Z))] \subseteq P$. To prove the second implication, let $x, y, z, R$ and $R^{\prime}$ be as in the premises of implication 2. It is sufficient to prove that $\operatorname{low}(x, R) \nsubseteq \operatorname{low}\left(x, R^{\prime}\right)$ or $(x, z) \in(Y \cup Z) \times Y$. Clearly by implication 1 , it follows that $z \in(Y \cup Z)$. If $z \in Y$, then evidently $(x, z) \in(Y \cup Z) \times Y$. So, suppose $z \notin Y$, which implies that $z \in Z$. Now because of $P \subseteq R$ and the definition of $P$, it follows that $(x, z) \notin P$. Of course $x \neq z$. So, there are $t \in A-(Y \cup Z)$ such that $x \in \operatorname{low}\left(t, R^{\prime}\right)$. Now, since $t \in A-(Y \cup Z)$, it follows that $y \ldots x \ldots t \ldots=R$. Thus, $\operatorname{low}(x, R) \nsubseteq$ low $\left(x, R^{\prime}\right)$. This proves the second implication and ends the proof of the claim.

Next we prove part (b). Let $L^{1}$ have an inseparable pair $(b, a)$. In view of Example 6, Theorem1 and $L^{1}$ being a maximal Maskin monotonic possibility domain, it follows for all $x \in A-\{b\}$ that $L_{x}(A) \subseteq L^{1}$ and $L_{b}^{1}=\{R \in L(A)$ : $b a \ldots=R\}$. So, for $(x, y) \neq(a, b)$, with $x \neq y$, there are $R \in L^{1}$ such that $y \ldots x=R$. Now, $(x, y) \in(Y \cup Z) \times Y$ would, by Maskin $\left(((Y \cup Z) \times Y) \cup I_{A}, L^{1}\right)$, imply that $\{x\} \times A \subseteq((Y \cup Z) \times Y)$ and therewith the contradiction $A \subseteq Y$. In consequence, $((Y \cup Z) \times Y)=\{(a, b)\}$. Hence, $\{a\}=Z$ and $\{b\}=Y$. This completes the proof of part (b). 
In order to prove (c) let $X_{1}$ and $X_{2}$ be a partition of $Y \cup Z$ with $\# X_{1} \geqslant 2$ and $X_{1} \times X_{2} \subseteq P$. It is sufficient to prove that this contradicts the maximality of $L^{N}$. Then $I_{A} \cup\left[X_{1} \times\left(Y \cap X_{1}\right)\right] \varsubsetneqq A \times A$ and $I_{A} \cup\left[X_{1} \times\left(Y \cap X_{1}\right)\right]$ is transitive. By the definition of $P$ it follows that $X_{1} \cap Y \neq \emptyset$. Consider $y \in$ $X_{1} \cap Y$ and $x \in X_{2}$. Then $(x, y) \in(Y \cup Z) \times Y$. Let $R \in L_{y}^{1}$ and let $R^{\prime} \in L(A)$ be such that $\left.R\right|_{A-\{x\}}=\left.R^{\prime}\right|_{A-\{x\}}$ and $y \ldots x=R^{\prime}$. Then $R^{\prime} \in L^{1}$ and $\operatorname{Maskin}\left(((Y \cup Z) \times Y) \cup I_{A}, L^{1}\right)$ would imply $\{x\} \times A \subseteq((Y \cup Z) \times Y)$ which then contradicts $Y \cup Z \neq A$. So, $R^{\prime} \notin L^{1}$. But obviously Maskin $\left(\left(X_{1} \times\right.\right.$ $\left.\left.\left(Y \cap X_{1}\right)\right) \cup I_{A}, L^{1} \cup\left\{R^{\prime}\right\}\right)$ which contradicts the maximality of $L^{N}$. Next we prove $(\mathrm{d})$.

First we prove $L^{1} \subseteq V$. Let $R \in L^{1}$. Then (1) follows because of the definition of $P$. In order to show that also (2) is satisfied let $z \ldots=R$. Let $T=$ (non-up $(z, P)-\{z\}) \cap(Y \cup Z)$. Take $x=\operatorname{best}\left(\left.R\right|_{T}\right)$. So $x \in T$ and for some $y \in Y$ there are $R^{\prime}$ in $L^{1}$ with $y \ldots z \ldots x \ldots=R^{\prime}$. Because of $z \in Z$, it follows that $(x, z) \notin((Y \cup Z) \times Y) \cup I_{A}$. Therefore Maskin $\left(((Y \cup Z) \times Y) \cup I_{A}, L^{1}\right)$ implies low $\left(x, R^{\prime}\right) \nsubseteq \operatorname{low}(x, R)$. So, there are $t \in \operatorname{low}\left(x, R^{\prime}\right)$ and $x \in \operatorname{low}(t, R)$. Because of the definition of $x$ and the fact that $x \in \operatorname{low}(t, R)$, it follows that $t \notin T$. But then, because $t \in \operatorname{low}\left(x, R^{\prime}\right)$, it follows that $t \in$ non$u p(z, P)-\{z\}$. Now, because $t \notin T$, it implies that $t \notin(Y \cup Z)$. So, $t \in A-(Y \cup Z)$ and $T \subseteq$ low $(t, R)$ which in turn yields (2).

Next we prove that $V \subseteq L^{1}$. Because of $L^{1} \subseteq V$ and the maximality of $L^{1}$, it is sufficient to prove that $V$ is a Maskin monotonic possibility domain. So, by Corollary 1 it is sufficient to prove that Maskin $\left(((Y \cup Z) \times Y) \cup I_{A}, V\right)$, which follows by the previous claim. This completes the proof of part $(d)$ and herewith the only if part.

(If-part) Let $Y, Z, P$ and $V$ as formulated in the if part of the theorem. Let $L^{1}=V$. It is sufficient to prove that $L^{N}$ is a Maskin monotonic possibility domain and that for all Maskin possibility domains $\widehat{L}^{N}$ with $V \subseteq \widehat{L}^{1}$ and $\widehat{L}^{i}=L(A)$, we have that $V=\widehat{L}^{1}$. Note that $P=\{(a, b) \in A \times A: \bar{a} \neq b$ and for all $y \in Y$ and all $\left.R \in L_{y}^{1} \ldots a \ldots b \ldots=R\right\}$. By Claim 1 we have Maskin $(((Y \cup$ $\left.Z) \times Y) \cup I_{A}, L^{1}\right)$. Setting $D$ of Lemma 1 equal to $((Y \cup Z) \times Y) \cup I_{A}$, it follows that $L^{N}$ is a Maskin monotonic possibility domain. Therefore it is sufficient to prove that there are no $I_{A} \varsubsetneqq D^{\prime} \varsubsetneqq A \times A$ and $V \varsubsetneqq W \subseteq L(A)$ with $\operatorname{Maskin}\left(D^{\prime}, W\right)$. To the contrary, let there be such $D^{\prime}$ and $W$. Without loss of generality we may assume that $W$ is a maximal Maskin monotonic possibility domain. By the only-if-part, we may assume the existence of disjoint $Y^{\prime}, Z^{\prime} \subseteq A$ with $Y^{\prime} \neq \emptyset, Y^{\prime} \cup Z^{\prime} \neq A$, an asymmetric and transitive relation $P^{\prime}$ on $A$ with $\left[\left(Y^{\prime} \cup Z^{\prime}\right) \times\left(A-\left(Y^{\prime} \cup Z^{\prime}\right)\right)\right] \subseteq P^{\prime} \subseteq\left[\left(Y^{\prime} \cup Z^{\prime}\right) \times\left(A-Y^{\prime}\right)\right]$, 
$\#\left(Y^{\prime} \cup Z^{\prime}\right) \geqslant 2$, if $L^{1}$ has a separable pair $(b, a)$ then $Y^{\prime}=\{b\}$ and $Z^{\prime}=\{a\}$, there is no partition $X_{1}^{\prime}, X_{2}^{\prime}$ of $Y^{\prime} \cup Z^{\prime}$ with $\# X_{1}^{\prime} \geqslant 2, X_{1}^{\prime} \times X_{2}^{\prime} \subseteq P^{\prime}$ and $W=\{R \in L(A):$

1. if $y \ldots=R$ for some $y \in Y^{\prime}$, then $P^{\prime} \subseteq R$;

2. if $z \ldots=R$ for some $z \in Z^{\prime}$, then there are $t^{\prime} \in A-\left(Y^{\prime} \cup Z^{\prime}\right)$ such that for all $x^{\prime} \in Y^{\prime} \cup Z^{\prime}$, with $x^{\prime} \neq z$ and $\left.\left(x^{\prime}, z\right) \notin P^{\prime}, x^{\prime} \in \operatorname{low}\left(t^{\prime}, R\right)\right\}$.

Now by Claim 1 it follows that Maskin $\left(\left(\left(Y^{\prime} \cup Z^{\prime}\right) \times Y^{\prime}\right) \cup I_{A}, W\right)$. Because of $V \subseteq W$, this implies Maskin $\left(\left(\left(Y^{\prime} \cup Z^{\prime}\right) \times Y^{\prime}\right) \cup I_{A}, V\right)$. Next we prove that $\left(\left(\left(Y^{\prime} \cup Z^{\prime}\right) \times Y^{\prime}\right) \cup I_{A}\right)=\left(((Y \cup Z) \times Y) \cup I_{A}\right)$.

First, we prove that $\left(\left(\left(Y^{\prime} \cup Z^{\prime}\right) \times Y^{\prime}\right) \cup I_{A}\right) \subseteq\left(((Y \cup Z) \times Y) \cup I_{A}\right)$. It is sufficient to prove that $Y^{\prime} \cup Z^{\prime} \subseteq Y \cup Z$ and that $Y^{\prime} \subseteq Y$. In order to prove the former let $x \in A-(Y \cup Z)$. It is sufficient to show that $x \notin Y^{\prime} \cup Z^{\prime}$. Clearly by the definition of $V$ it follows that $L_{x}(A)=V_{x}$. Because of $V \subseteq W$, it follows that $V_{x} \subseteq W_{x}$. Hence, $L_{x}(A)=W_{x}$. Suppose $x \in Y^{\prime}$. Then there are $z \in\left(Y^{\prime} \cup Z^{\prime}\right)-\{x\}$ and $R \in L_{x}(A)=W_{x}$ such that $x \ldots z=R$. Now by the assumptions on $Y^{\prime}, Z^{\prime}, P^{\prime}$ and $W$ we have that $\left(Y^{\prime} \cup Z^{\prime}\right) \times\left(A-\left(Y^{\prime} \cup Z^{\prime}\right)\right) \subseteq P \subseteq R$. As $x \ldots z=R, x \in Y^{\prime}$ and $z \in$ $Y^{\prime} \cup Z^{\prime}$ this can only hold if $A=Y^{\prime} \cup Z^{\prime}$ which contradicts our assumptions on $Y^{\prime}, Z^{\prime}, P^{\prime}$ and $W$. Suppose $x \in Z^{\prime}$. Then there are $R \in L_{x}(A)=W_{x}$ such that $\left(Y^{\prime} \cup Z^{\prime}\right) \times\left(A-\left(Y^{\prime} \cup Z^{\prime}\right)\right) \subseteq R$. Now as there are $t \in A-\left(Y^{\prime} \cup Z^{\prime}\right)$ such that $\left(\left(\right.\right.$ non-up $\left.\left.\left(x, P^{\prime}\right)-\{x\}\right) \cap\left(Y^{\prime} \cup Z^{\prime}\right)\right) \subseteq$ low $(t, R)$ it follows that ((non$\left.\left.u p\left(x, P^{\prime}\right)-\{x\}\right) \cap\left(Y^{\prime} \cup Z^{\prime}\right)\right)=\emptyset$. As there is no partition $X_{1}^{\prime}$ and $X_{2}^{\prime}$ of $Y^{\prime} \cup Z^{\prime}$ such that $X_{1}^{\prime} \times X_{2}^{\prime} \subseteq P^{\prime}$ and $\# X_{1}^{\prime} \geq 2$ we have $Y^{\prime}=\{y\}$ and $Z^{\prime}=\{x\}$. Hence $(y, z)$ is an inseparable pair in $W$ and therewith as well in $V$. This however leads by (b) to the contradiction $Y=Y^{\prime}=\{y\}$ and $Z=Z^{\prime}=\{x\}$.

In order to prove $Y^{\prime} \subseteq Y$ let $y \in Y^{\prime}$. To the contrary assume $y \notin Y$. Then by the inclusion $Y^{\prime} \cup Z^{\prime} \subseteq Y \cup Z$ we may conclude that $y \in Z$ and that there are $t \in(A-(Y \cup Z)) \subseteq\left(A-\left(Y^{\prime} \cup Z^{\prime}\right)\right)$. Furthermore, there are $R \in V_{y}$ with $y t \ldots=R$. As $V \subseteq W$, it follows that $R \in W_{y}$ where $y \in Y^{\prime}$. But $y t \ldots=R$ with $t \in A-\left(Y^{\prime} \cup Z^{\prime}\right)$ contradicts $\left[\left(Y^{\prime} \cup Z^{\prime}\right) \times\left(A-\left(Y^{\prime} \cup Z^{\prime}\right)\right)\right] \subseteq P \subseteq R$. Hence, $Y^{\prime} \subseteq Y$.

Next we prove $\left(((Y \cup Z) \times Y) \cup I_{A}\right) \subseteq\left(\left(\left(Y^{\prime} \cup Z^{\prime}\right) \times Y^{\prime}\right) \cup I_{A}\right)$. By the proof of the previous inclusion, we may assume that both $\left(Y^{\prime} \cup Z^{\prime}\right) \subseteq(Y \cup Z)$ and $Y^{\prime} \subseteq Y$.

First we show that $Y=Y^{\prime}$. To the contrary let $b \in Y-Y^{\prime}$. Now for all $c \in Y^{\prime} \subseteq Y$, there are preferences $R$ and $R^{\prime}$ in $V$ such that $c b \ldots=R$ and 
$b c \ldots=R^{\prime}$. If $b \notin\left(Y^{\prime} \cup Z^{\prime}\right)$, then $R \in V \subseteq W$ violates condition 1 of $W$. So, $b \in\left(Y^{\prime} \cup Z^{\prime}\right)$ which means that $b \in Z^{\prime}$. Because of $R \in W$, it follows that $(x, b) \notin P^{\prime}$ for all $x \in A-\{c, b\}$. Now, because $R^{\prime} \in W$,in view of condition 2 of $W$ it follows that $(c, b) \in P^{\prime}$. Note that for all $x \in Y-\{b, c\}$, there are preferences $R^{\prime \prime}=x b c \ldots$ in $V \subseteq W$. So, because of $(c, b) \in P^{\prime}$, it follows that $Y^{\prime}=\{c\}$. Since $\{c, b\} \subseteq Y$, it follows that $(c, b)$ is not an inseparable pair in $V$. Therefore, there are $x \in A-\{c, b\}$ and preferences $R^{\prime \prime \prime}=c x \ldots b \ldots$ in $V \subseteq W$. Then Maskin $\left(\left(\left(Y^{\prime} \cup Z^{\prime}\right) \times Y^{\prime}\right) \cup I_{A}, W\right)$ implies $x \in\left(Y^{\prime} \cup Z^{\prime}\right)$. As $Y^{\prime}=\{c\}$ this yields $x \in Z^{\prime}$. Because of $R^{\prime \prime \prime} \in V$ and $c \in Y$, it follows that $(y, x) \notin P$ for all $y \in A-\{c, x\}$. But then, there are preferences $R^{\prime \prime \prime \prime}=b c x \ldots$ in $V \subseteq W$. Note that although $b, x \in Z^{\prime}$ and $(x, b) \notin$ $P^{\prime}$, there is no $t^{\prime} \in A-\left(Y^{\prime} \cup Z^{\prime}\right)$ such that $b \ldots t^{\prime} \ldots x \ldots=R^{\prime \prime \prime \prime}$ contradicting condition 2 of $W$. In consequence, $Y=Y^{\prime}$.

In the following, we prove that $Y \cup Z=Y^{\prime} \cup Z^{\prime}$. To the contrary let $Y \cup Z \neq Y^{\prime} \cup Z^{\prime}$. We will prove that $\left[\left(Y^{\prime} \cup Z^{\prime}\right) \times\left((Y \cup Z)-\left(Y^{\prime} \cup Z^{\prime}\right)\right)\right] \subseteq P$ which because of $\#\left(Y^{\prime} \cup Z^{\prime}\right) \geqslant 2$ clearly contradicts the assumptions on $P$. So let $x^{\prime} \in\left(Y^{\prime} \cup Z^{\prime}\right)$ and let $z \in\left((Y \cup Z)-\left(Y^{\prime} \cup Z^{\prime}\right)\right)$. Because of $Y=Y^{\prime}$ it follows that $z \in Z-Z^{\prime}$. Suppose to the contrary that $\left(x^{\prime}, z\right) \notin P$. Then there are $y^{\prime} \in Y$ and $R \in V$ with $y^{\prime} \ldots z \ldots x^{\prime} \ldots=R$. As $Y=Y^{\prime}$ both $x^{\prime}$ and $y^{\prime}$ are in $\left(Y^{\prime} \cup Z^{\prime}\right)$ Maskin $\left(\left(\left(Y^{\prime} \cup Z^{\prime}\right) \times Y^{\prime}\right) \cup I_{A}, W\right)$ implies the contradiction $z \in\left(Y^{\prime} \cup Z^{\prime}\right)$. So, $\left(x^{\prime}, z\right) \in P$ and herewith $\left[\left(Y^{\prime} \cup Z^{\prime}\right) \times\left((Y \cup Z)-\left(Y^{\prime} \cup Z^{\prime}\right)\right)\right] \subseteq$ $P$.

So, $Y=Y^{\prime}$ and $Z=Z^{\prime}$. Next we show that $P=P^{\prime}$ which then by the definition of $V$ and $W$ yields the desired result that $V=W$. First we show that $P \subseteq P^{\prime}$. To the contrary, suppose that $(a, b) \in P$ and $(a, b) \notin P^{\prime}$. Because $P \cup P^{\prime} \subseteq[(Y \cup Z) \times(A-Y)]$ and $((Y \cup Z) \times(A-(Y \cup Z))) \subseteq P \cap P^{\prime}$, it follows that $a \in Y \cup Z$ and $b \in Z$. Now because of the definition of $V$ there are $R \in V$ with bat... $=R$ for some $t \in(A-(Y \cup Z))$. Since $V \subseteq W$, this clearly yields a contradiction with condition 2 of $W$. So, $P \subseteq P^{\prime}$.

Next we prove $P^{\prime} \subseteq P$. Suppose $(a, b) \notin P$. We prove that $(a, b) \notin P^{\prime}$. Because $(a, b) \notin P$ it follows by the definition of $V$ that there are $R \in V$ such that both $y \ldots=R$ for some $y \in Y$ and ...b...a... $=R$. As $R \in V \subseteq W$. This shows that $(a, b) \notin P^{\prime}$. 


\section{Conclusion}

By restricting the domain of only one agent, we showed that it is possible to escape the negative conclusions of the GS and the MS theorems. Obviously, the rules that are non-dictatorial, Pareto efficient and strategy-proof/Maskin monotonic have a dictatorship flavor since, at many preference profiles, the agent with a restricted set of preferences gets his top alternative. A open question of interest would be to study how these rules evolve as we restrict the preferences of more than one agent. We close the discussion with two examples showing that tackling this question may be a non-trivial hurdle. The first example shows that if we restrict the sets of preferences of exactly two agents, then neither the condition of inseparable pair nor the condition of inseparable set is a necessary condition for the maximality of a strategy-proof possibility domain.

Example 7 Let $A=\{w, x, y, z\}$ and $N=\{1,2\}$. Take $L^{1}=\{R \in L(A)$ : $R=x \ldots$ or $R=y \ldots x \ldots z \ldots$ or $R=w \ldots x \ldots z \ldots$ or $R=z \ldots\}$ and $L^{2}=\{R \in$ $L(A): R=x \ldots z \ldots w \ldots$ or $R=y \ldots w \ldots x \ldots$ or $R=z \ldots x$ or $R=w \ldots y \ldots x \ldots\}$. Now define the rule $K$ as follows. For each $p \in L^{N}$,

$$
K(p)= \begin{cases}a & \text { if } p(1)=a \ldots \text { and } p(2)=b \ldots \text { and }(a, b) \notin\{(y, x),(w, x)\} \\ x & \text { otherwise. }\end{cases}
$$

So at $K$ agent 2 is decisive on the pairs $(x, y)$ and $(x, w)$ whereas agent 1 is decisive on the remaining pairs. So $K$ is not dictatorial. As $K(p)$ $\in\{\operatorname{best}(p(1))$, best $(p(2))\}$ we have that $K$ is Pareto efficient. To see that $K$ is strategy-proof consider profiles $p$ with $p(2)=x \ldots$. Then the outcome for any $\{1\}$-deviation of $p$ is either $x$ or $z$ depending on whether agent 1 prefers $x$ to $z$ or $z$ to $x$ respectively. A similar reasoning holds for $\{2\}$-deviation of profile $p$ with $p(1)=y \ldots$ or $p(1)=w \ldots$. It is straight forward to see that for both agents $i$ the set $L^{i}$ does not have an inseparable pair or set. By which we may conclude that these inseparabilities are not necessary conditions for maximal strategy-proof possibility domains in case the set of preferences of more than one agent is restricted. Note further that $K$ is tops-only.

The following and last example show a strategy-proof possibility domain for two agents with the same set of admissible preferences. Also in this case these sets are not containing an inseparable pair or set. Moreover the social choice rule which is discussed in this example is not tops-only. 
Example 8 Let $A=\{w, x, y, z\}$ and $N=\{1,2\}$. Take $L^{1}=L^{2}=\{R \in$ $L(A): R=x y w z$ or $R=x w \ldots$ or $R=y z w x$ or $R=y w \ldots$ or $R=z x w y$ or $R=z w \ldots$ or $R=w \ldots\}$. Note that at every profile there is at most one alternative different from $w$ which is strictly preferred to it by both agent 1 and 2 simultaneously.

Let rule $K$ be defined as follows. For each $p \in L^{N}$,

$$
K(p)= \begin{cases}a & \text { if there exists a such that } p(1)=. . a . . w . . \text { and } p(2)=. . a . . w . . \\ w & \text { otherwise. }\end{cases}
$$

Since this rule treats agents symmetrically, it is non-dictatorial. Given the special position of $w, K$ is also Pareto efficient. In general such imputation social choice correspondences on unrestricted domains are strategy-proof if the outcome sets are compared on their best alternatives only. But then as $K$ yields a singleton at each of the profiles in the restricted domain at hand, we have as a consequence that it is strategy-proof. Notice that this set of admissible preferences does neither have an inseparable pair nor an inseparable set.

\section{References}

1. Aswal N., Chatterji S., Sen A., 2003, "Dictatorial Domains," Economic Theory, 22, p. 45-62.

2. Barbera S., Gul F., Stacchetti E., 1993, "Generalized Median Voter Schemes and Committees," Journal of Economic Theory, 61, p. 262289.

3. Barbera S., Massó J., Neme A., 1997, "Maximal Domains of Preferences Preserving Strategy-Proofness for Generalized Median Voter Schemes," Social Choice and Welfare, 16, p.298-321.

4. Barbera S., Sonnenschein H., Zhou L., 1991, "Voting by Committees," Econometrica, 59, p. 595-609.

5. Bochet O., Klaus B., 2008, "The Relation between Monotonicity and Strategy-Proofness," Mimeo

6. Ching S., Serizawa S., 1998, "A Maximal Domain for the Existence of Strategy-Proof Rules," Journal of Economic Theory, 78, 157-166. 
7. Gibbard A., 1973, "Manipulation of Voting Schemes: A general result," Econometrica, 41, p. 587-601.

8. Gibbard A., 1977, "Manipulation of Schemes that Mix Voting and Chance," Econometrica, 45, p. 665-681.

9. Gibbard A., 1978, "Straightforwardness of Game Forms with Lotteries as Outcomes," Econometrica, 46, p. 595-614.

10. Kalai E. and Muller E., 1977, "Characterization of Domains Admitting Nondictatorial Social Welfare Functions and Nonmanipulable Voting Procedures," Journal of Economic Theory, 16, p.457 469.

11. Kalai E. and Ritz Z., 1980, "Characterization of the Private Alternatives Domains Admitting Arrow Social Welfare Functions," Journal of Economic Theory, 22, p.23 36.

12. Massó J., Neme A., 2001, "A Maximal Domain of Preferences for the Division Problem," Games and Economic Behavior, 37, p.367-387.

13. Moulin H., 1980, "On Strategy-proofness and Single Peakedness," Public Choice, 35, p.437-455.

14. Peters H., v.d. Stel H., Storcken T., 1991, "On Uncompromisingness and Strategy-proofness," Research Memorandum, M91-15, Maastricht University.

15. Puppe C., Tasnádi A., 2007, "Nash Implementable Domains for the Borda Count," Forthcoming in Social Choice and Welfare.

16. Satterthwaite M.A., 1975, "Strategy-proofness and Arrow's conditions: Existence and Correspondence Theorem for Voting Procedures and Social Welfare Functions," Journal of Economic Theory, 10,p.187-217.

17. Serizawa S., 1995, "Power of Voters and Domain of Preferences where Voting by Committees is Strategy-proof," Journal of Economic Theory, 67, p.599 608 .

18. Storcken T., 1989, Possibility Theorems for Social Welfare Functions, Tilburg University. 
19. Zhou L., 1990, "Impossibility of Strategy-proof Mechanisms in Economies with Pure Public Goods," Review of Economic Studies, 58, p.107-119.

\section{Appendix}

Proposition 3 Let $K: L^{N} \rightarrow A$ be a social choice function. Then

1. $K$ is strategy-proof if and only if it is intermediate strategy-proof;

2. If $K$ is strategy-proof, then it is Maskin monotonic.

Proof. (First part) Clearly intermediate strategy-proofness implies strategyproofness. So, suppose $K$ is strategy-proof. Furthermore, let $p$ and $q$ be $S$-deviations for some $S \subseteq N$ such that $p(i)=R$ for some $R \in L(A)$. It is sufficient to proof that $\ldots K(q) \ldots K(p) \ldots \neq R$. Without loss of generality let $S=\{1,2, \ldots, s\}$. Take profiles $r^{0}, r^{1}, r^{2}, \ldots, r^{s} \in L^{N}$ defined for all $i \in N$ by $r^{t}(i)=p(i)$ if $i>t$, and by $r^{t}(i)=q(i)$ otherwise. So, $r^{0}=p$ and $r^{s}=q$. By strategy-proofness, it follows that $\ldots K\left(r^{t+1}\right) \ldots K\left(r^{t}\right) \ldots \neq r^{t}(t+1)=R$ for all $t \in\{0,1,2, \ldots, s-1\}$. Because of negative transitivity of $R$, it follows that $\ldots K(q) \ldots K(p) \ldots \neq R$.

(Second part) Let $p$ and $q$ be $\{i\}$-deviations in $L^{N}$ such that,

$$
\operatorname{low}(K(p), p(i)) \subseteq \operatorname{low}(K(p), q(i)) .
$$

It is sufficient to prove that $K(p)=K(q)$. By strategy-proofness it follows that $K(q) \in \operatorname{low}(K(p), p(i))$ and $K(p) \in$ low $(K(q), q(i))$. Given our assumption, we have that $K(q) \in \operatorname{low}(K(p), q(i))$ and $K(p) \in \operatorname{low}(K(q), q(i))$. As $q(i)$ is antisymmetric we have that $K(p)=K(q)$. 